\title{
Research on Fused Tapered Photonic Crystal Fiber Sensor Based on the Method of Intermittent Cooling
}

\author{
Guangwei Fu, Xinghu Fu, Peng Guo, Yushen Ji, and Weihong Bi \\ The Key Laboratory for Special Fiber and Fiber Sensor of Hebei Province, School of Information Science and Engineering, \\ Yanshan University, Qinhuangdao 066004, China \\ Correspondence should be addressed to Guangwei Fu; earl@ysu.edu.cn and Weihong Bi; bwhong@ysu.edu.cn
}

Received 24 August 2016; Accepted 21 September 2016

Academic Editor: Lei Yuan

Copyright (C) 2016 Guangwei Fu et al. This is an open access article distributed under the Creative Commons Attribution License, which permits unrestricted use, distribution, and reproduction in any medium, provided the original work is properly cited.

Based on the intermittent cooling method, a fused tapered Photonic Crystal Fiber (PCF) interferometer is proposed. In the process of tapering, stop heating and wait for cooling at different taper length. Repeat heating and cooling, until taper goes to the expected length. Compared with the ordinary fused tapered method, the fringe contrast of the transmission spectra of this sensor is $15.06 \mathrm{~dB}$. The transmission spectra in different concentrations of glycerol solution are obtained, and the temperature cross-sensitivity of the sensor is studied. The experimental results show that as the external refractive index increases, the transmission spectra of the sensor shift to longer wavelength. In the measuring glycerol solution, the refractive index sensitivity of the sensor can achieve $797.674 \mathrm{~nm} / \mathrm{RIU}$, and the temperature sensitivity is only $0.00125 \mathrm{~nm} /{ }^{\circ} \mathrm{C}$.

\section{Introduction}

Photonic Crystal Fiber (PCF) [1-3] is a new kind of optical fiber, with different structures and optical transmission characteristics from single-mode fiber. The cladding region is composed of microholes arraying paralleled to the axial direction of the fiber. Therefore, PCF has strong flexible structure design, which would open up a new area of the production and application of optical fiber device, such as no deadline single-mode transmission, large and effective field area, high nonlinearity, and high birefringence.

Taper technique of PCF can change the internal structure and optical properties of PCF, with important potential value to PCF device production and the exploration of application in the sensing field $[4,5]$. Liu et al. [6] for the first time tapered the PCF and made research on optical soliton self-frequency shift. After that research about the theory, fabrication and application of tapered PCF became the focus of scholars studies. Leon-Saval et al. [7] proposed a "rapid low temperature" method to taper PCF and then to maximally control air holes collapse. Jasim et al. [8] took the measure of flame heating to stretch two compact taper areas, and the current sensitivity of Mach-Zehnder sensor could be up to $40.26 \mathrm{pm} / \mathrm{A}^{2}$. And there was a report [9] about an M-Z interferometer using SMF-PCF-SMF structure with conventional technique for refractive index measurement and the maximum refractive index sensitivity as of $198.77 \mathrm{~nm} / \mathrm{RIU}$. In addition, the optical properties [10-12], the optical waveguide coupling [13], and the generation of supercontinuum characteristics of tapered PCF $[14,15]$ are widely studied by many scholars.

Based on conventional tapering technique, an intermittent cooling fused tapered is adopted to make a kind of interferometer sensor with larger fringe contrast, and the refractive index sensing characteristics are analyzed; the refraction index sensitivity of the glycerin aqueous solution used in detection is up to $797.674 \mathrm{~nm} / \mathrm{RIU}$. Compared to conventional taper technology, its sensitivity has been greatly improved.

\section{The Theoretical Analysis}

Fused tapering technology is to put the fiber without coating into high temperature flame and then stretch the fused fiber on both sides at the same time. Finally, a special waveguide with tapered structure is formed in the heating area to 


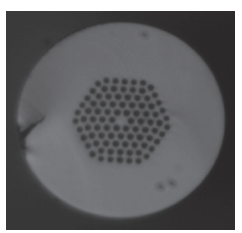

(a) Fiber cross section

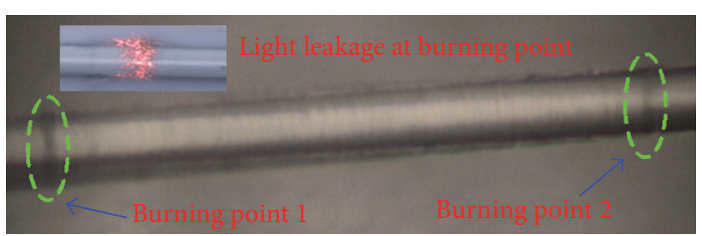

(b) Light source free burning point

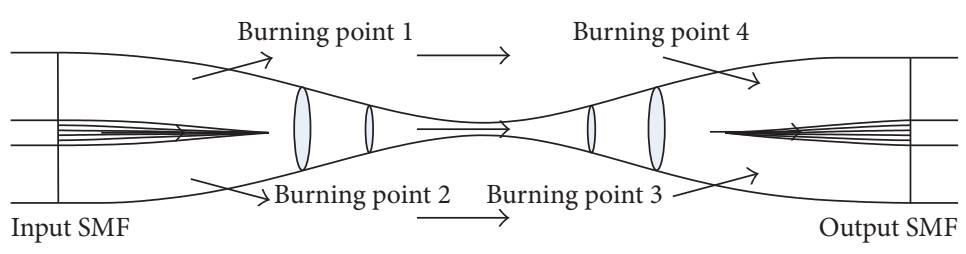

(c) Schematic diagram of the sensing structure

FIGURE 1: Fused tapered PCF sensor.

transmit light. As beam transmitting through the tapered area, there are two processes that are beam splitting and beam combining, respectively. And then the whole light path forms as a Mach-Zehnder interferometer. The technology of intermittent cooling is to suspend the tapering process at different tapering length and continue tapering after cooling. By means of this method, the ignition points are formed in tapered area. Because the change of refractive index in ignition point area causes the mode-mismatched phenomenon, part of light in the core will leak into cladding again. This light-leaking phenomenon leads to the cladding mode's effective refractive index being more susceptible to the outside refractive index. So it can improve the refractive index sensitivity.

Through the method of intermittent cooling, a section of PCF is stretched as tapered structure. The fused tapered PCF sensor is shown in Figure 1.

The PCF used in the experiment has the advantage of infinite single-mode transmission. And when PCF is connected with single-mode fiber by fiber fusion splicer, try not to make the PCF's air holes collapse by adjusting the fusion splicing parameters. So, as beam passing through the first ignition point, the light coupling into the PCF can still transmit in the PCF's fiber core. And only the fundamental mode is transmitted.

It is supposed that there exist four ignition points. When light transmits to tapered area, core mode transforms to cladding mode gradually with the diameter of tapered area decreasing regularly. Light energy transmitting as core mode reduces slowly and cladding mode is stimulated correspondingly. When light transmits through the first ignition point, part of light will leak into cladding, and the same situation will occur as light transmits to the next three ignition points. With the diameter of tapered area at the output end increase by degrees, the light energy transmitting as cladding mode reduces gently, and the fundamental mode enhances correspondingly. When light transmits to nontapered area, cladding mode couples into fiber core and interferes with fundamental mode of fiber core. The interference intensity and central wavelength can be expressed as

$$
\begin{aligned}
I & =I_{1}+I_{2}+2 \sqrt{I_{1} I_{2}} \cos \varphi \\
\lambda_{m} & =\frac{\Delta n_{\mathrm{eff}} L}{m}
\end{aligned}
$$

In (1), $I$ is the total output light intensity; $I_{1}$ and $I_{2}$ are light intensities of the fundamental mode and cladding mode, respectively; $\varphi$ is phase displacement; $\lambda_{m}$ is the central wavelength of level $m$; $L$ is the interference length, which is the distance between two fused points; and $\Delta n_{\text {eff }}$ is the difference between core's refractive index and cladding mode's effective refractive index.

When environmental refractive index changes, the cladding mode effective refractive index changes correspondingly, while the core refractive index remains constant. The wavelength shift caused by the change of outside refractive index is expressed as

$$
\Delta \lambda_{m}=\frac{\left(\Delta n_{\mathrm{eff}}+\Delta n\right) L}{m}-\frac{\Delta n_{\mathrm{eff}} L}{m}=\frac{\Delta n L}{m} .
$$

In (2), $\Delta \lambda_{m}$ is the central wavelength shift of level $m ; \Delta n$ is the variation of refractive index difference caused by the change of environmental refractive index.

It can be seen from (2) that when interference length $L$ is a constant, the interference fringe's wavelength shift changes linearly with the change of environmental refractive index. So the environmental refractive index could be measured by detecting the central wavelength shift of level $m$.

Because of the light leak at ignition points, the energy of cladding mode has been greatly enhanced, and the interference is more obvious. At the same time, the light carrying outside information enters into optical fiber, and the coupling degree between sensing area and outside environment is enhanced further. So, the sensitivity of sensor is improved. 


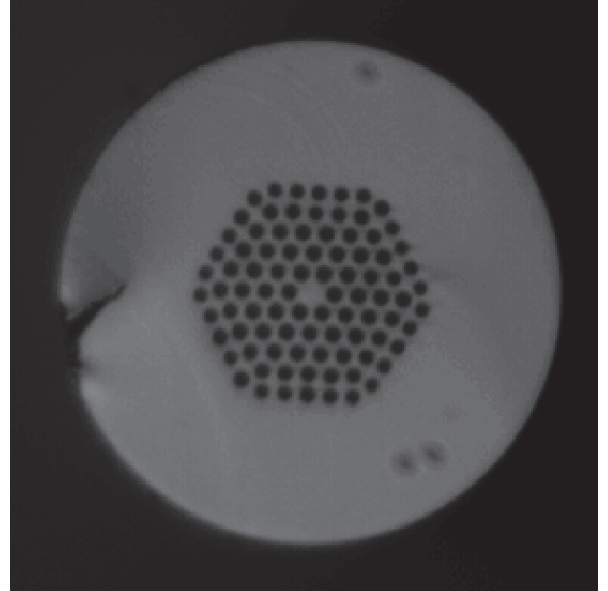

(a) Diameter of $125 \mu \mathrm{m}$

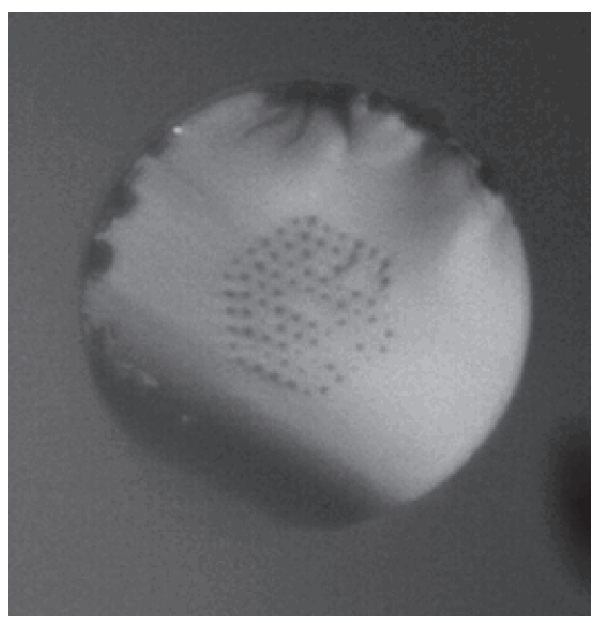

(c) Diameter of $55.37 \mu \mathrm{m}$

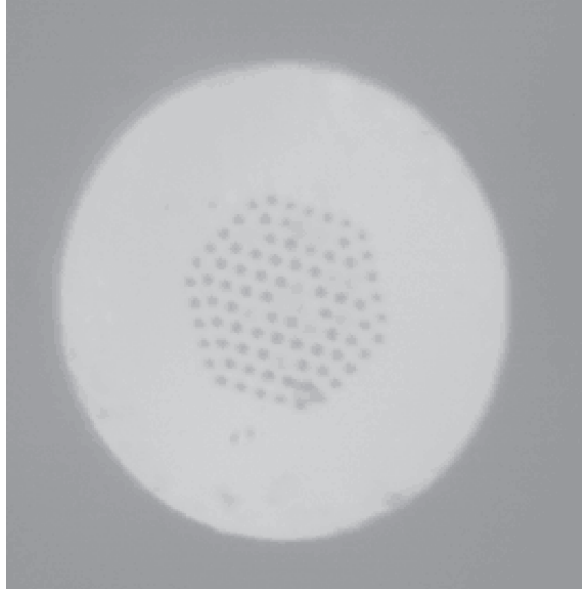

(b) Diameter of $98.72 \mu \mathrm{m}$

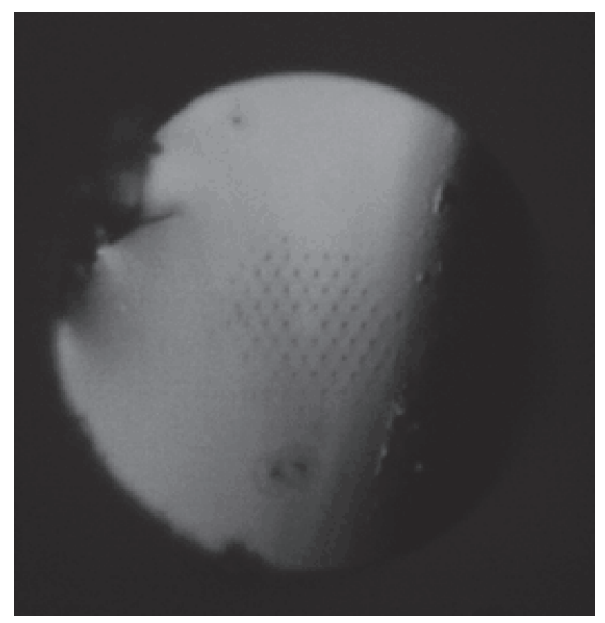

(d) Diameter of $32.86 \mu \mathrm{m}$

FIGURE 2: Cross sections of different diameters.

\section{Experimental and Results}

3.1. Sensor Production. The used PCF in the lab is SM-7 solid core PCF, the outer cladding layer diameter is $125 \mu \mathrm{m}$, the core diameter is $7.0 \mu \mathrm{m}$ with 5 layers of air hole and a hexagon structure arrangement, the air hole diameter is about $2.63 \mu \mathrm{m}$, and the hole spacing is about $4.22 \mu \mathrm{m}$.

The used splicing machine is FITEL S178 optical fiber fusion splicer, optical taper machine is SCS-4000 optical fused taper system, light source is ASE broadband light source $(1520 \mathrm{~nm}-1610 \mathrm{~nm})$, and AQ6317B spectrometer is used to detect the sensor spectrum.

In the process of fabrication, first of all, it should adjust the parameter of fusion splicer and make a sensor with SMFPCF-SMF structure, where length of PCF is $26 \mathrm{~mm}$. Then the optical taper machine is used to fusedly taper the PCF, when the length is $1 \mathrm{~mm}, 3 \mathrm{~mm}$, and $7 \mathrm{~mm}$ in turn, break off heating and tapering, till it is cool and then start heating and tapering. In the overall taper process, fiber is fixed in the tensile platform, so that the fiber cannot move at all to ensure the heating zone does not change. When the taper length reaches $12 \mathrm{~mm}$, tapering process will be over. PCF taper region length is $12 \mathrm{~mm}$; the taper additional loss is $12.56 \mathrm{~dB}$. Different diameters of taper region result in different cross sections of fiber, as shown in Figure 2.

3.2. Glycerin Aqueous Solution Sensing Experiment. The configuration concentration of glycerin aqueous solution is $5 \%$ r $25 \%$, and Abbe refractometer is used to measure the refractive index of solution; the refractive index changes in the range of 1.342 1.379.

Put tapered PCF sensor into the sample pool, mix glycerin aqueous solution with different concentrations, until the tapered PCF sensor completely immersed into the solution, and then measure transmission spectra. Before measuring, PCF taper region must be cleaned with distilled water every time. ASE light source output power is $16 \mathrm{dBm}$. The experimental system is shown in Figure 3.

The experimental measurement results of transmission spectra corresponding with different concentrations of glycerol aqueous solution are shown in Figure 4. 


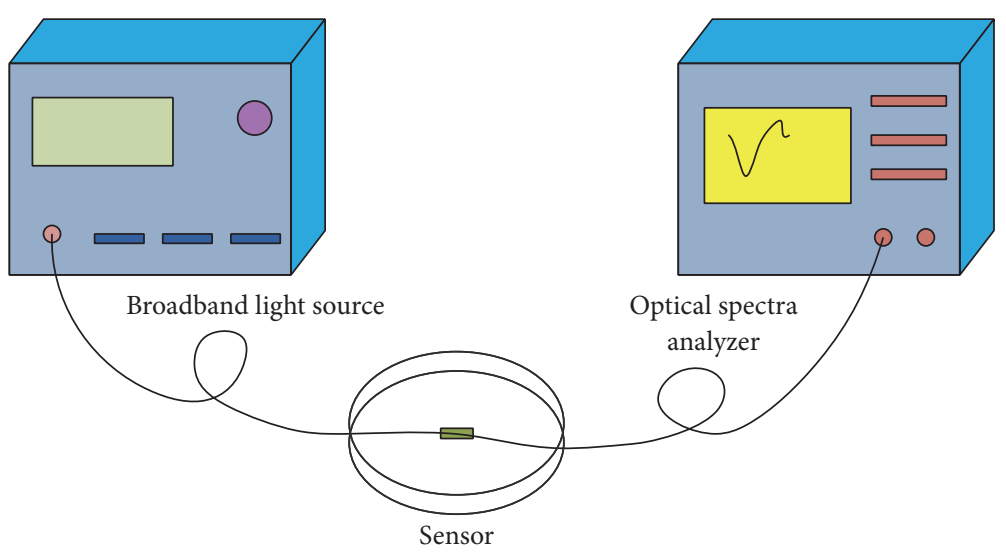

FIGURE 3: Schematic of the fused tapered PCF sensor system.

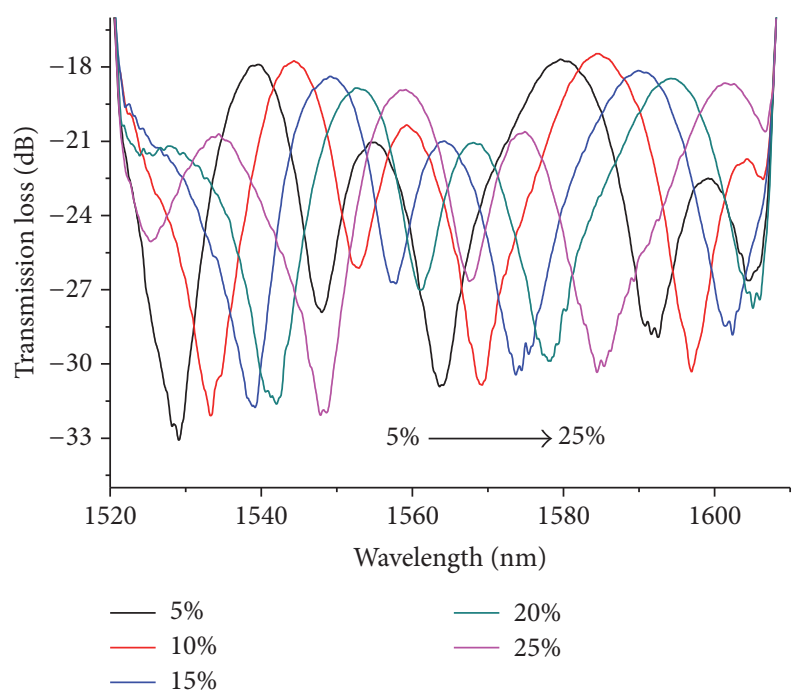

FIGURE 4: Transmission spectrum of the fused tapered PCF sensor.

In Figure 4, it can be seen that the transmission spectra have significant shifts to the long wave direction, with the increasing of concentration of glycerol solution. The peaks change within the vicinity of $1590 \mathrm{~nm}$ wavelength. The details of the spectrum are as shown in Figure 5.

As shown in Figure 5, it can be found that with the increasing of the concentration of the transmission, the spectrum is shifted to longer wavelength. As the solution concentration ranges from $5 \%$ to $25 \%$, wavelength drifts over $20 \mathrm{~nm}$. When repeating the experiment and making data fitting, the relationship between wavelength shift and the refractive index of solution can be obtained, as shown in Figure 6.

In Figure 6, with the increase of the refractive index of solution, the interference fringe center wavelength drifts to long wavelength direction a lot, and there is a good linear relationship; the sensitivity of the refractive index is $797.674 \mathrm{~nm} / \mathrm{RIU}$.

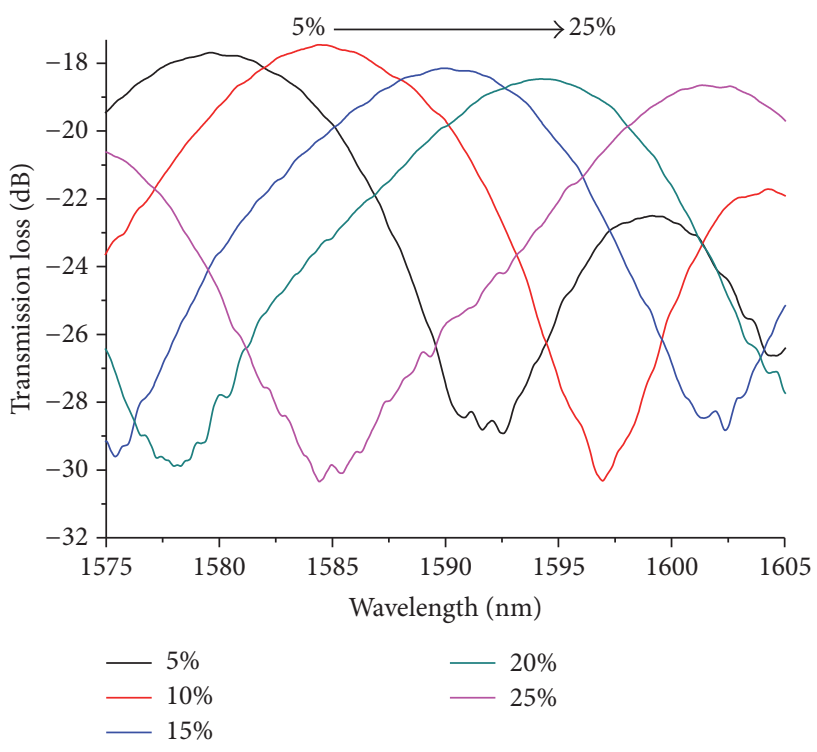

FIGURE 5: Transmission spectra of the tapered PCF sensor in different liquids at wavelength of $1590 \mathrm{~nm}$.

3.3. Temperature Sensing Experiment. Put the PCF sensor into the temperature controlled oven and connect both ends to ASE light source $(1520 \sim 1610 \mathrm{~nm})$ and the spectrometer, respectively. ASE light source output power is $16 \mathrm{dBm}$. Schematic diagram of the temperature sensing experimental system is as shown in Figure 7.

Heat the temperature controlled oven from $20^{\circ} \mathrm{C}$ to $70^{\circ} \mathrm{C}$ and measure it every $10^{\circ} \mathrm{C}$. Detect the transmission spectrum with OSA when the temperature is stable. The same measurement is done in the opposite temperature changing as it declines from $70^{\circ} \mathrm{C}$ to $20^{\circ} \mathrm{C}$. The result of the measurement is shown in Figure 8.

It can be seen from Figure 8 that the overall trend of the transmission spectra remains unchanged at different temperature. Study the wave crest at the region about $1597.90 \mathrm{~nm}$ and make the data linear fitting; a relationship between the 


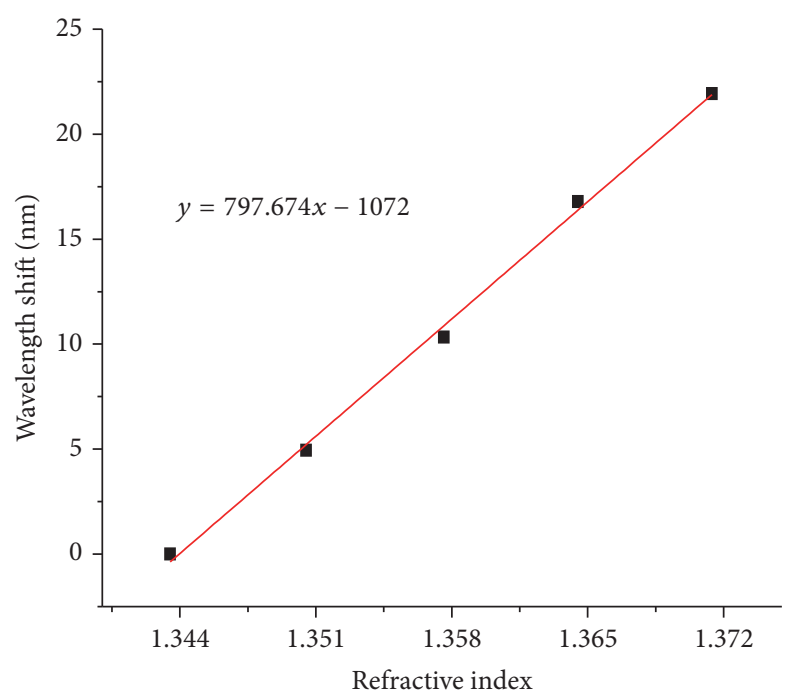

FIGURE 6: Relationship between wavelength shift and refractive index.

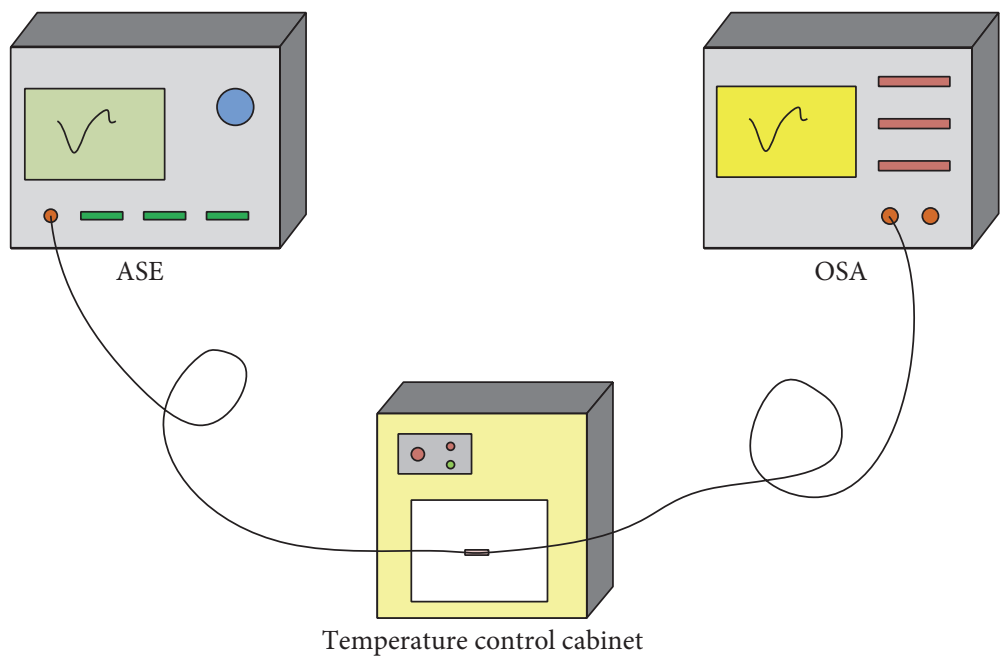

FIgURE 7: The temperature sensing system.

transmission spectrum wavelength shift of the tapered PCF and the outside temperature can be obtained, as shown in Figure 9.

It can be viewed that the transmission spectrum of the PCF sensor shifts gently towards long wavelength direction when the outside temperature is changed, according to Figure 9. The temperature sensitivity is merely $0.00125 \mathrm{~nm} /{ }^{\circ} \mathrm{C}$ indicating that the intermittent cooling fused tapered PCF is not sensitive to temperature. Considering that temperature can induce the central wavelength shift of level $m$ for transmission spectrum of interferometer, it can be known according to [16] that the relationship between interference central wavelength and temperature can be expressed as $\Delta \lambda_{m}^{\prime}=\left(\alpha+P_{t}\right) \lambda_{m} \Delta T$, in which $\alpha$ is the coefficient of thermal expansion about the materials of interferometer; for pure $\mathrm{SiO}_{2}$, it is $5 \times 10^{-7} /{ }^{\circ} \mathrm{C}$. $P_{t}$ is the variation of effective refractive index difference between two interference patterns caused by temperature change: $P_{t}=(1 / \Delta n) \partial(\Delta n) / \partial T$, where $\Delta T$ represents temperature change. Because both of the fiber core mode and the cladding mode in tapered PCF can transfer in undoped PCF, intervening with temperature on the influence of the fiber core and cladding mode is the same; thus $P_{t}=0$. And the thermal expansion coefficient, $\alpha=5 \times 10^{-7} /{ }^{\circ} \mathrm{C}$, owns a really small numerical value. Therefore, $\Delta \lambda_{m}^{\prime} \approx 0$, which is identical to the experiment results obtained from Figures 8 and 9. To sum up, it can be concluded that the intermittent cooling fused tapered sensor interferometer is not sensitive to temperature.

\section{Conclusion}

This paper presents using an intermittent cooling fused taper method to produce an interferential Photonic Crystal Fiber (PCF) sensor. To fulfill PCF fused tapering in the process, intermittent cooling in the melting process is introduced. Compared with the ordinary fused taper, multiple times of 


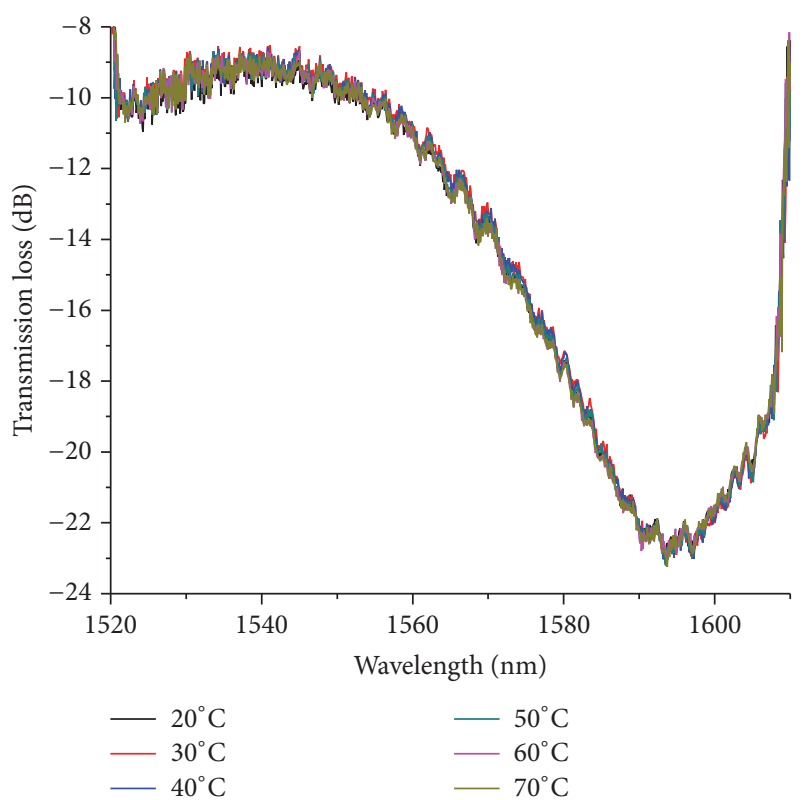

Figure 8: Transmission spectrum of the fused tapered PCF sensor with different temperature.

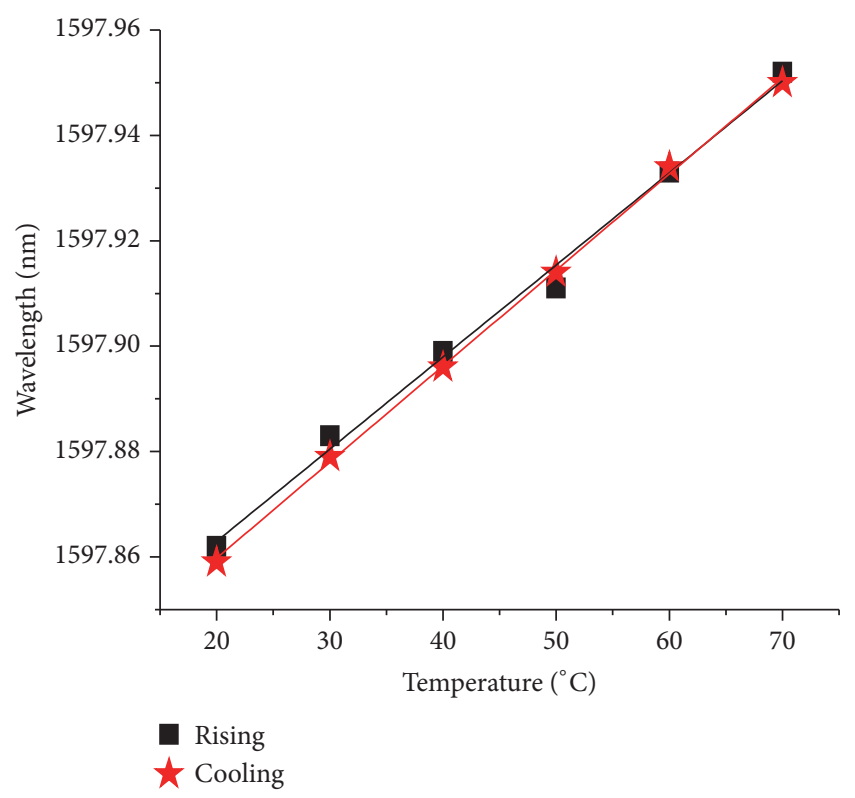

FIGURE 9: Relationship between wavelength shift and temperature.

manufacturing taper PCF sensor have larger fringe visibility. Further research is done to study the sensor response to external environment with different refractive index. The experimental results show that, when immersed the taper PCF sensor in the different concentration solution environment, along with the increasing external refractive index, the center wavelength significantly drifts to long wavelength direction. The refractive index sensitivity measured in aqueous glycerol solution is up to $797.674 \mathrm{~nm} / \mathrm{RIU}$. Compared with taper PCF produced by ordinary fused taper method, the sensitivity is greatly improved. At the same time, the temperature sensitivity of the sensor is only $0.00125 \mathrm{~nm} /{ }^{\circ} \mathrm{C}$, which can be considered to be insensitive to temperature, and it can overcome the cross-sensitivity problem of the simultaneous measurement for refractive index and temperature.

\section{Competing Interests}

The authors declare that there is no conflict of interests regarding the publication of this paper.

\section{Acknowledgments}

This project is supported by the National Natural Science Foundation of China (nos. 61575170, 61475133, and 61675176), the Key Applied Basic Research Program of Hebei Province (nos. 16961701D and QN2016078), Hebei Provincial Natural Science Foundation (nos. F2015203270 and F2016203392), Qinhuangdao Science and Technology Support Program (no. 201601B050), College Youth Talent Project of Hebei Province (no. BJ2014057), and "XinRuiGongCheng" Talent Project of Yanshan University.

\section{References}

[1] J. C. Knight, T. A. Birks, P. S. J. Russell, and D. M. Atkin, "Allsilica single-mode optical fiber with photonic crystal cladding," Optics Letters, vol. 21, no. 19, pp. 1547-1549, 1996.

[2] T. A. Birks, J. C. Knight, and P. S. J. Russell, "Endlessly singlemode photonic crystal fiber," Optics Letters, vol. 22, no. 13, pp. 961-963, 1997.

[3] P. S. J. Russell, "Photonic-crystal fibers," Journal of Lightwave Technology, vol. 24, no. 12, pp. 4729-4749, 2006.

[4] E. C. Mägi, P. Steinvurzel, and B. J. Eggleton, "Tapered photonic crystal fibers," Optics Express, vol. 12, no. 5, pp. 776-784, 2004.

[5] H. C. Nguyen, B. T. Kuhlmey, E. C. Magi et al., "Tapered photonic crystal fibres: properties, characterization, and applications," in Proceedings of the Micro-Technologies for the New Millennium International Society for Optics and Photonics Conference, vol. 5840, pp. 29-43, Sevilla, Spain, May 2005.

[6] X. Liu, C. Xu, W. H. Knox et al., "Soliton self-frequency shift in a short tapered air-silica microstructure fiber," Optics Letters, vol. 26, no. 6, pp. 358-360, 2001.

[7] S. G. Leon-Saval, T. A. Birks, W. J. Wadsworth, P. S. J. Russell, and M. W. Mason, "Supercontinuum generation in submicron fibre waveguides," Optics Express, vol. 12, no. 13, pp. 2864-2869, 2004.

[8] A. A. Jasim, S. W. Harun, M. Z. Muhammad, H. Arof, and H. Ahmad, "Current sensor based on inline microfiber MachZehnder interferometer," Sensors and Actuators, A: Physical, vol. 192, no. 7, pp. 9-12, 2013.

[9] C.-P. Tang, M. Deng, T. Zhu, and Y.-J. Rao, "Photonic crystal fiber based $\mathrm{M}-\mathrm{Z}$ interferometer for refractive index measurement," Journal of Optoelectronics Laser, vol. 22, no. 9, pp. 13041308, 2011.

[10] H. C. Nguyen, B. T. Kuhlmey, M. J. Steel et al., "Leakage of the fundamental mode in photonic crystal fiber tapers," Optics Letters, vol. 30, no. 10, pp. 1123-1125, 2005.

[11] B. T. Kuhlmey, H. C. Nguyen, M. J. Steel, and B. J. Eglleton, "Confinement loss in adiabatic photonic crystal fiber tapers," 
Journal of the Optical Society of America B: Optical Physics, vol. 23, no. 9, pp. 1965-1974, 2006.

[12] X. Xi, Z. Chen, G. Sun, and J. Hou, "Mode-field expansion in photonic crystal fibers," Applied Optics, vol. 50, no. 25, pp. E50E54, 2011.

[13] J. G. Liu, T.-H. Cheng, Y.-K. Yeo et al., "Light beam coupling between standard single mode fibers and highly nonlinear photonic crystal fibers based on the fused biconical tapering technique," Optics Express, vol. 17, no. 5, pp. 3115-3123, 2009.

[14] J. M. Dudley and S. Coen, "Coherence properties of supercontinuum spectra generated in photonic crystal and tapered optical fibers," Optics Letters, vol. 27, no. 13, pp. 1180-1182, 2002.

[15] G. Humbert, W. J. Wadsworth, S. G. Leon-Saval et al., "Supercontinuum generation system for optical coherence tomography based on tapered photonic crystal fibre," Optics Express, vol. 14, no. 4, pp. 1596-1603, 2006.

[16] U. Møller, S. T. Sørensen, C. Larsen et al., "Optimum PCF tapers for blue-enhanced supercontinuum sources," Optical Fiber Technology, vol. 18, no. 5, pp. 304-314, 2012. 


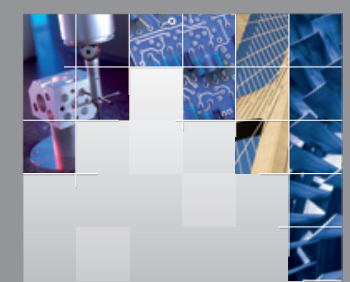

\section{Enfincering}
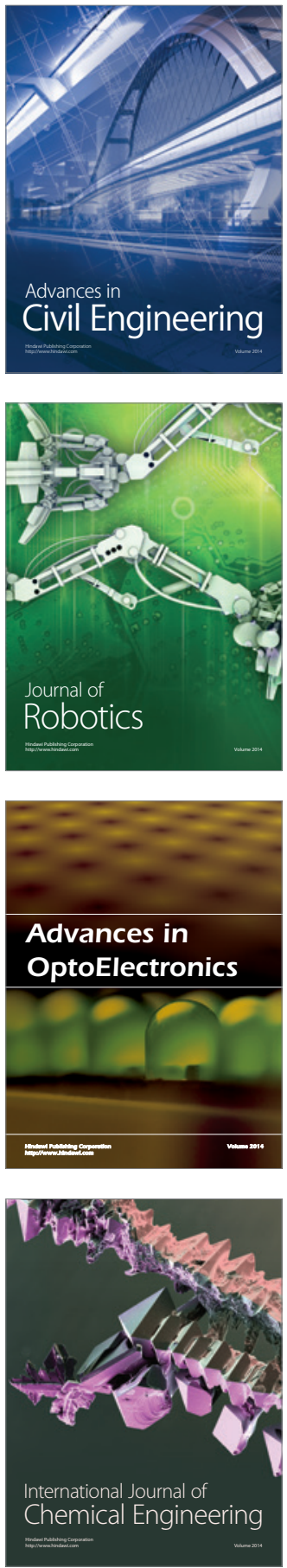

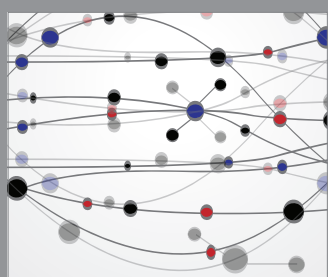

The Scientific World Journal

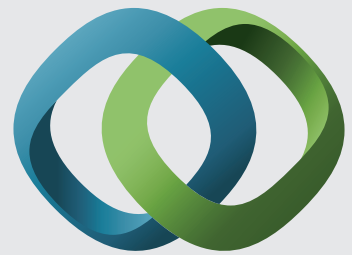

\section{Hindawi}

Submit your manuscripts at

http://www.hindawi.com
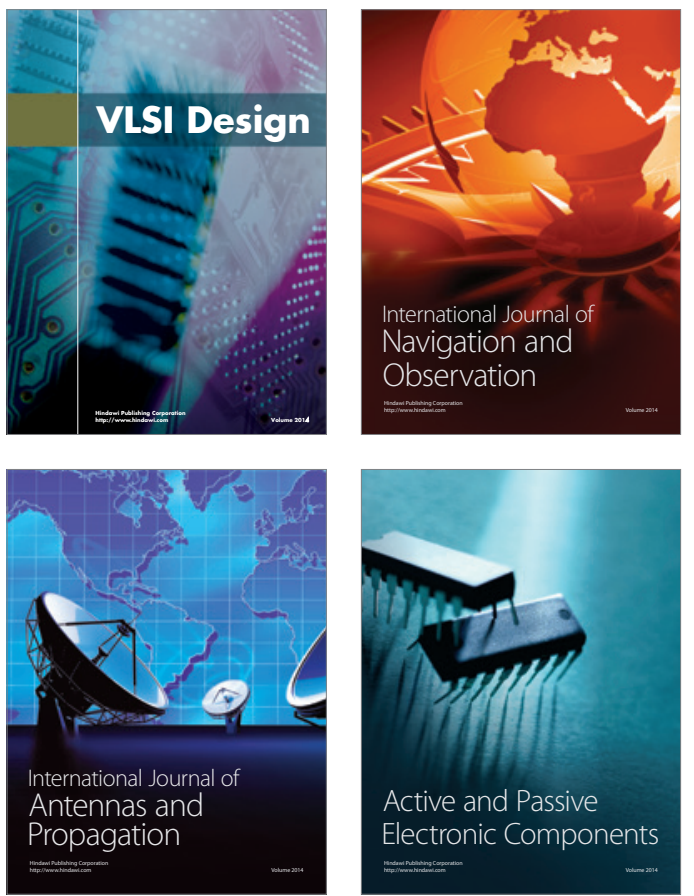
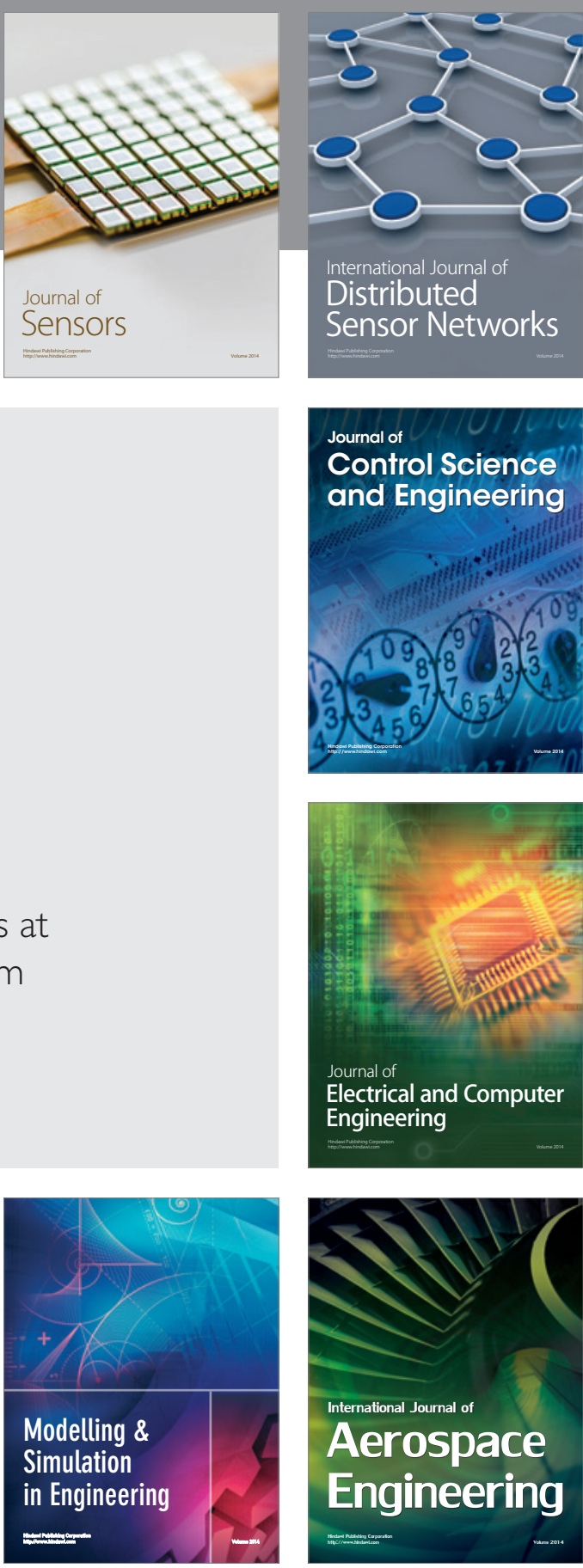

International Journal of

Distributed

Sensor Networks

Journal of

Control Science

and Engineering
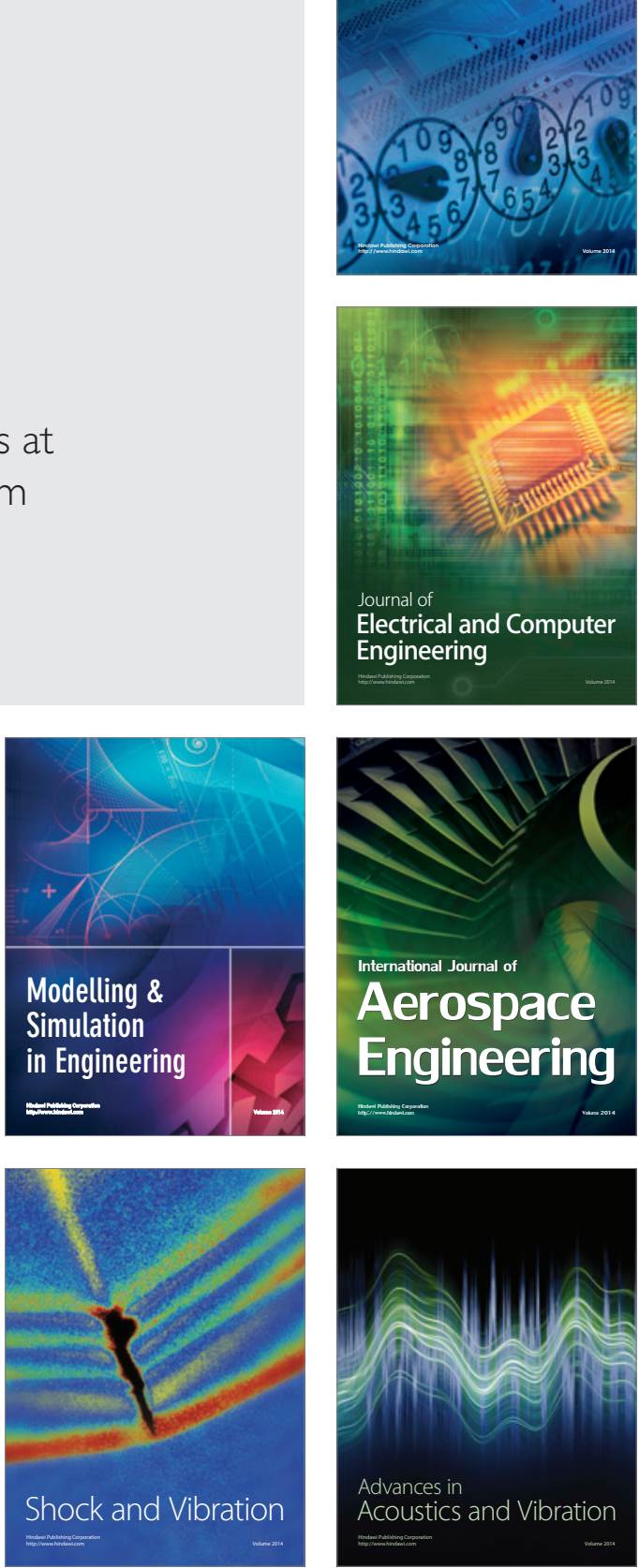